\title{
Control of the Polarization of a Vacuum-Ultraviolet, High-Gain, Free-Electron Laser
}

\author{
Enrico Allaria, ${ }^{1}$ Bruno Diviacco, ${ }^{1}$ Carlo Callegari, ${ }^{1}$ Paola Finetti, ${ }^{1}$ Benoît Mahieu, ${ }^{2}$ Jens Viefhaus, ${ }^{3}$ Marco Zangrando, \\ Giovanni De Ninno, ${ }^{1,5}$ Guillaume Lambert, ${ }^{2}$ Eugenio Ferrari, ${ }^{1,6}$ Jens Buck, $^{7}$ Markus Ilchen, ${ }^{7,8}$ Boris Vodungbo, ${ }^{9,10}$ \\ Nicola Mahne, ${ }^{1}$ Cristian Svetina, ${ }^{1,11}$ Carlo Spezzani, ${ }^{1}$ Simone Di Mitri, ${ }^{1}$ Giuseppe Penco, ${ }^{1}$ Mauro Trovó, ${ }^{1}$ \\ William M. Fawley, ${ }^{1}$ Primoz R. Rebernik, ${ }^{1,5}$ David Gauthier, ${ }^{1,5}$ Cesare Grazioli, ${ }^{5,1,4}$ Marcello Coreno, ${ }^{1,12}$ Barbara Ressel, ${ }^{5}$ \\ Antti Kivimäki, ${ }^{4}$ Tommaso Mazza, ${ }^{7}$ Leif Glaser, ${ }^{3}$ Frank Scholz, ${ }^{3}$ Joern Seltmann, ${ }^{3}$ Patrick Gessler, ${ }^{7}$ Jan Grünert, ${ }^{7}$ \\ Alberto De Fanis, ${ }^{7}$ Michael Meyer, ${ }^{7}$ André Knie, ${ }^{13}$ Stefan P. Moeller, ${ }^{14}$ Lorenzo Raimondi, ${ }^{1}$ Flavio Capotondi, ${ }^{1}$ \\ Emanuele Pedersoli, ${ }^{1}$ Oksana Plekan, ${ }^{1}$ Miltcho B. Danailov, ${ }_{1}^{1}$ Alexander Demidovich, ${ }^{1}$ Ivaylo Nikolov, ${ }^{1}$ \\ Alessandro Abrami, ${ }^{1}$ Julien Gautier, ${ }^{2}$ Jan Lüning, ${ }^{9,10}$ Philippe Zeitoun, ${ }^{2}$ and Luca Giannessi, ${ }^{1,15}$ \\ ${ }^{1}$ Elettra-Sincrotrone Trieste, Basovizza I-34149, Italy \\ ${ }^{2}$ Laboratoire d'Optique Appliquée, ENSTA ParisTech-CNRS UMR 7639-Ecole polytechnique, \\ Chemin de la Hunière, 91761 Palaiseau, France \\ ${ }^{3}$ DESY, FS-PE, Notkestrasse 85, 22607 Hamburg, Germany \\ ${ }^{4}$ CNR Istituto Officina dei Materiali, Laboratorio TASC, 34149 Basovizza, Trieste, Italy \\ ${ }^{5}$ Laboratory of Quantum Optics, University of Nova Gorica, Ajdovščina 5270, Slovenia \\ ${ }^{6}$ Dipartimento di Fisica, Università degli Studi di Trieste, Piazzale Europa 1, 34127 Trieste, Italy \\ ${ }^{7}$ European XFEL, Albert-Einstein-Ring 19, 22761 Hamburg, Germany \\ ${ }^{8}$ Stanford PULSE Institute, 2575 Sand Hill Road, Menlo Park, California 94025, USA \\ ${ }^{9}$ Sorbonne Universités, UPMC Paris 06, UMR 7614, LCPMR, 75005 Paris, France \\ ${ }^{10}$ CNRS, UMR 7614, LCPMR, 75005 Paris, France \\ ${ }^{11}$ Graduate School of Nanotechnology, University of Trieste, Piazzale Europa 1, 34127 Trieste, Italy \\ ${ }^{12}$ CNR-ISM, UOS RM1, CP10, I-00019 Monterotondo Scalo (RM), Italy \\ ${ }^{13}$ Universität Kassel, Institut für Physik, Heinrich-Plett-Straße 40, 34132 Kassel, Germany \\ ${ }^{14}$ SLAC National Accelerator Laboratory, 2575 Sand Hill Road, Menlo Park, California 94025, USA \\ ${ }^{15}$ ENEA, Via Enrico Fermi, 45, 00044 Frascati Roma, Italy
}

(Received 20 June 2014; revised manuscript received 20 August 2014; published 2 December 2014)

The two single-pass, externally seeded free-electron lasers (FELs) of the FERMI user facility are designed around Apple-II-type undulators that can operate at arbitrary polarization in the vacuum ultraviolet-to-soft x-ray spectral range. Furthermore, within each FEL tuning range, any output wavelength and polarization can be set in less than a minute of routine operations. We report the first demonstration of the full output polarization capabilities of FERMI FEL-1 in a campaign of experiments where the wavelength and nominal polarization are set to a series of representative values, and the polarization of the emitted intense pulses is thoroughly characterized by three independent instruments and methods, expressly developed for the task. The measured radiation polarization is consistently $>90 \%$ and is not significantly spoiled by the transport optics; differing, relative transport losses for horizontal and vertical polarization become more prominent at longer wavelengths and lead to a non-negligible ellipticity for an originally circularly polarized state. The results from the different polarimeter setups validate each other, allow a cross-calibration of the instruments, and constitute a benchmark for user experiments.

DOI: 10.1103/PhysRevX.4.041040

\section{INTRODUCTION}

Circularly polarized light is a critical tool to investigate many important properties of matter. In atomic and molecular physics, circular polarization is directly associated with angular-momentum selection rules, resulting in

Published by the American Physical Society under the terms of the Creative Commons Attribution 3.0 License. Further distribution of this work must maintain attribution to the author $(s)$ and the published article's title, journal citation, and DOI.
Subject Areas: Interdisciplinary Physics,

Particles and Fields, Photonics ent angular distribution of photoelectrons [1]). Circular dichroism can be used to directly probe the chirality of a system, which may stem from geometry, typically in biomolecules [2], or from magnetic properties. In the latter case, spin-orbit interactions provide a handle to access and manipulate these properties with optical photons on the femtosecond time scale [3]. At X-ray wavelengths, circular polarized light has also been used to produce highresolution, holographic images of magnetic domains [4] and to study the time evolution of the magnetization 
recovery in order to distinguish the different time scales of the spin and orbital angular momenta [5]. As a final example, polarization of the incoming or outgoing radiation can be directly related to the three-dimensional electron wave function through recently developed methods of electron tomography [6] and angular streaking [7].

Up to and including the near-UV range, materials and methods for generating and measuring circular polarization are well developed and affordable, with a multitude of applications [8]. At higher photon energies ranging up to hard $\mathrm{x}$ rays, variable polarization undulators have been utilized in synchrotron light sources to provide circularly polarized light [9-13], albeit at time scales that are typically much longer (i.e., tens of ps or longer) and at peak brightnesses much below those provided in linear polarization by the new generation of soft and hard $\mathrm{x}$-ray free-electron lasers (FELs) [14] such as FLASH [15], LCLS [16], and SACLA [17]. These machines were designed and currently operate with linearly polarized undulators for multiple reasons, including the desire for high magnetic fields at short undulator periods to maximize the FEL gain and lower construction cost. Linearly polarized undulators are also believed to have smaller error fields than those generally found in variable gap, variable polarization undulators of the Apple-II type [18] and related designs [19].

However, there remains a strong need for intense, femtosecond time-scale, short-wavelength radiation with variable polarization [20]. With such light, fields such as time-resolved $\mathrm{x}$-ray magnetic circular dichroism (XMCD) spectroscopy can explore ultrafast magnetization dynamics. The femto-slicing technique [21] on synchrotron light sources has produced circularly polarized, hard x-ray ultrashort pulses but with quite limited peak power and total pulse energy. In the soft $\mathrm{x}$-ray spectral range, highharmonic-generation sources (HHG), previously thought to be limited to only linearly polarized emission, may be manipulated to produce elliptically polarized light [22]. Here too, though, both the peak power and total pulse energy are extremely small compared to what is commonly produced by the aforementioned FEL sources such as LCLS and SACLA. Although methods for partial control of the output $\mathrm{x}$-ray polarization for these nominally linearly polarized sources by means of either polarizer filters [23] or via crossed-field undulators [24,25] have been proposed and in part demonstrated, in principle [26,27], none has been operationally implemented yet; both SACLA and LCLS are working toward possible solutions that would allow users to choose the FEL polarization. Very recently, by means of specially designed diamond-crystal optics that can be used in the hard $\mathrm{x}$ ray as a phase retarder, the SACLA team has measured a high degree of circular and also vertically polarized radiation at about 1 angstrom [28]. A special Delta-type undulator is under construction [29] and is to be placed at the end of LCLS to generate $\mathrm{x}$-ray radiation with a significant degree of circular polarization.
FERMI $[30,31]$ is the first operational, externally seeded FEL facility designed for user experiments. Based on the high-gain harmonic generation scheme (HGHG) [32], FERMI produces 100-fs duration, 100-MW to multi-GW peak intensity radiation pulses in the vacuum ultraviolet (VUV)-to-soft $\mathrm{x}$-ray spectral range that are characterized by an unprecedented photon energy stability and a high degree of longitudinal and transverse coherence [33,34]. A crucial difference that distinguishes FERMI from other high-power, short-wavelength FEL user facilities is its use of variable-gap Apple-II undulators, thus giving users the ability to vary and control both the wavelength and the polarization of the emitted light on time scales of minutes or less. Over the past year, users at FERMI have already taken advantage of these unique capabilities, e.g., to study dichroic effects via atomic photo-ionization [35] and coherent imaging in ferromagnetic materials [36-38].

Inasmuch the actual purity of the output polarization is both an important quantity for many users and, in principle, can be degraded by undulator magnetic topology errors and, given the high-gain environment, possibly also electron-beam trajectory errors, we conducted a systematic characterization of the polarization states of FERMI's FEL-1 radiation. This effort involved a dedicated experiment that employed three different and independent methods to measure the output radiation polarization over a resonant wavelength range extending from 26 to $54 \mathrm{~nm}$. Our results constitute the first systematic characterization of the polarization states produced by a high-gain, highpeak-power FEL operating in the VUV spectral range with variable polarization undulators. At each measured wavelength, all the experimental diagnostics confirm that, as expected from standard single-particle emission theory [39], FERMI's output polarization state may be controlled and adjusted directly via the undulator polarization. Our findings are in accordance with earlier polarization measurements made on visible and UV FEL light, both for a storagering FEL [40] and for low-gain, single-pass seeded FELs $[27,41-43]$ where a very high degree of polarization was found both at the resonant FEL wavelength and at the higher harmonics [42]. They also agree with a recent FERMI user experiment [35] that provided independent evidence at a single VUV wavelength $(25.6 \mathrm{~nm})$ of a similar high degree of polarization for the output light of FERMI's FEL-1.

The remainder of this paper is organized as follows. First, in Sec. II, we briefly describe the FERMI FEL system used in this experiment. Section III reports a description of the three independent polarimeters used. Section IV presents the results obtained from each polarimeter and their analysis. We finish with a general discussion of the measurements and with our conclusions.

\section{FERMI's FEL-1}

The dedicated polarization experiment was performed at FEL-1, the first of two FELs in operation at FERMI 
TABLE I. Parameters characterizing the electron beam used in the experiment.

\begin{tabular}{lcc}
\hline \hline Energy & 1.22 & $\mathrm{GeV}$ \\
Slice energy spread (rms) & 150 & $\mathrm{keV}$ \\
Slice transverse normalized emittance & $\approx 1$ & $\mathrm{~mm} \mathrm{mrad}$ \\
Peak current & 600 & $\mathrm{~A}$ \\
Average beam transverse size & 150 & $\mu \mathrm{m}$ \\
$\quad$ in the undulator (rms) & & \\
Repetition rate & 10 & $\mathrm{~Hz}$ \\
\hline \hline
\end{tabular}

[30,31]. FERMI is a FEL user facility designed to operate in the VUV soft $\mathrm{x}$-ray spectral range with two FEL lines that share the same electron accelerator [44]. FEL-1 covers the longer-wavelength spectral range (100-20 nm), while FEL-2 can reach wavelengths as short as $4 \mathrm{~nm}$ by means of a double-stage HGHG configuration [34]. Both FELs use the electron beam accelerated by a normal-conducting, S-band radio-frequency (RF) linac, whose final electronbeam energy can be varied in the range $0.9-1.5 \mathrm{GeV}$; a detailed description of the linac can be found in Ref. [45]. For the reported experiment, the accelerator has been operated to produce an electron beam whose characteristics are reported in Table I. The FERMI FEL-1 HGHG undulator system is sketched in Fig. 1 and described in Sec. II B; the value of its main parameters, specific to this experiment, are reported in Table III.

The HGHG process is initiated by the interaction between the electron beam and the external seed laser, occurring in the first undulator (modulator). The interaction gives rise to beam-energy modulation and bunching at the harmonics of the seed-laser wavelength. This bunching promotes coherent emission at the desired harmonic, which is further amplified by the FEL gain process occurring in the successive six undulator segments (radiators) (see Fig. 1).

\section{A. The seed laser}

Two modes of operation are possible for the FERMI seed-laser system, both driven by a fixed-wavelength (783 nm) Ti:Sapphire laser. When continuous tunability of the FEL wavelength is needed, the Ti:Sapphire pumps an optical parametric amplifier (OPA) [46]. This is the configuration used with the fluorescence polarimeter (Sec. III D), where the FEL wavelength must match an atomic resonance $(53.7 \mathrm{~nm}$, corresponding to the 5 th harmonic of the seed OPA tuned to $268.5 \mathrm{~nm}$ ). If fixedseed-wavelength operation is sufficient, the third harmonic

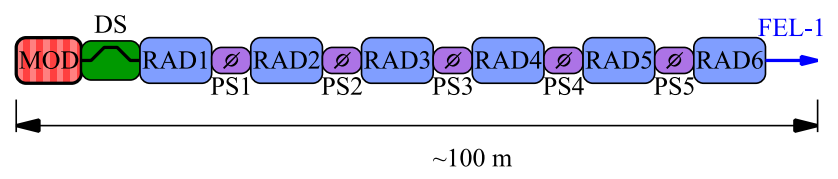

FIG. 1. Schematic layout of the FEL-1 undulator system. See text for details.
TABLE II. Seed-laser parameters used in this work for the two possible modes of operation (THG and OPA).

\begin{tabular}{lccl}
\hline \hline & THG & OPA & \\
\hline Wavelength & 261 & 268.5 & $\mathrm{~nm}$ \\
Bandwidth & 0.8 & 1.2 & $\mathrm{~nm}$ \\
Pulse length (FWHM) & 150 & 180 & $\mathrm{fs}$ \\
Energy per pulse & 10 & 10 & $\mu \mathrm{J}$ \\
Average beam size & 300 & 300 & $\mu \mathrm{m}$ \\
$\quad$ in the undulator (rms) & & & \\
\hline \hline
\end{tabular}

TABLE III. Main parameters for the FERMI FEL-1 undulators. The tuning range is estimated for the electron-beam energy used in the experiment, i.e., $1.2 \mathrm{GeV}$.

\begin{tabular}{lccc}
\hline \hline & Modulator & Radiators & \\
\hline Magnetic period & 100 & 55 & $\mathrm{~mm}$ \\
$\begin{array}{l}\text { Tuning range } \\
\begin{array}{l}\text { Maximum undulator } \\
\quad \text { deflection parameter (rms) }\end{array}\end{array}$ & $300-100$ & $70-20$ & $\mathrm{~nm}$ \\
$\begin{array}{l}\text { Number of periods } \\
\quad \text { per segment }\end{array}$ & 30 & 3.7 & \\
$\begin{array}{l}\text { Number of segments } \\
\text { Polarization }\end{array}$ & 1 & 42 & \\
& Horizontal & $\begin{array}{l}\text { Elliptical } \\
\text { (adjustable) }\end{array}$ \\
\hline \hline
\end{tabular}

of the Ti:Sapphire laser at $261 \mathrm{~nm}$ is used (THG configuration) and affords some extra flexibility on seed-laser power and other seed-laser parameters. This is the configuration used with the other polarimeters (Secs. III B and III C). The nominal seed-laser parameters for the two configurations are summarized in Table II.

\section{B. The undulators}

The system consists of a fixed-polarization permanentmagnet modulator (MOD), an electromagnetic dispersive section (DS), and six permanent-magnet variably polarized radiators (RAD1, 2, ., 6). The 3-m-long modulator, based on a standard Halbach-array configuration [47], is tuned so that its resonant wavelength matches that of the seed laser, by adjusting the vertical gap between the upper and the lower magnet arrays.

The following dispersive section is a three-pole wiggler providing a wide range of field strengths. This section is needed in order to guarantee that the energy-modulated electron beam develops the optimum charge-density modulation (bunching) required for different operational conditions of the FEL (i.e., different FEL harmonics and different levels of seed-laser power). The radiators are Apple-II-type undulators [18], allowing variable wavelength and continuously adjustable elliptical polarization by means of a concerted movement (vertical gap and longitudinal shift) of the four magnetic arrays. For this 
experiment, however, the nominal polarizations were set to be either strictly circular or strictly linear; at any given wavelength, the typical time required to switch the polarization was of the order of one minute. The six radiators are separated by $1.3-\mathrm{m}$ breaks, where quadrupoles, steerers, and diagnostic stations (beam-position and transverse beam-profile monitors) are accommodated.

In addition, a series of permanent-magnet phase-matching units (PS1, 2, ...) [48] guarantees the correct phase relationship (i.e., constructive interference) between successive radiators, so that the six undulator segments (each $2.4 \mathrm{~m}$ long) behave essentially as a single, 14-m-long device. Taking advantage of the seeding process, the available undulator length is sufficient to allow the FEL to reach saturation with a typical pulse energy of several tens of $\mu \mathrm{J}$.

\section{MEASUREMENT SETUP}

\section{A. The photon transport system}

At the exit of the final radiator undulator, the FEL pulses enter the photon-beam-transport system (PADReS) that conveys them to the experimental stations and provides for their characterization. PADReS includes a diagnostic ensemble that can determine online and on a shot-to-shot basis the intensity, the divergence, the position, and the spectral content of each radiation pulse [49]. Since these diagnostics are completely noninvasive, in all three experiments, these parameters were acquired in parallel to the polarimeter data, together with all the most relevant electronbeam parameters. All this information was stored digitally by the real-time FERMI control system [50], tagged by the corresponding unique bunch number for each FEL shot.

After the diagnostic section (which also includes two plane mirrors and the energy spectrometer grating), the photons were transported towards the end stations diffraction and projection imaging (DiProI) and low density matter (LDM) (Fig. 2), where they could be routed into the three different polarimeters installed for this experiment. Along the optical path of PADReS, several optical elements affect the photon beam. The elements common to both beam lines are as follows: an angle-defining aperture, the above-mentioned set of plane mirrors, and the diffraction grating (used in zeroth order). A vertical-deflecting mirror and a set of Kirkpatrick-Baez (KB) refocusing mirrors [51,52] are installed along each of the beam lines. All the optics work in grazing-incidence geometry; their main parameters are reported in Table IV.

Photon fluence to the experiment was controlled by insertable Al filters located at several locations along the optical path and by a gas absorber cell that can be filled with an absorbing gas (currently $\mathrm{N}_{2}$ ) [49].

The polarimeters were physically mounted on different end stations (e-TOF and VUV-optical polarimeters on DiProI, the fluorescence polarimeter on LDM). It is therefore important to note that the optical paths to the two end stations differ: After the common part, comprising three horizontal reflections (PM1a, PM1 b, and LE grating), the beam sent to LDM undergoes an additional horizontal reflection (LDM switching), which is not required when reaching DiProI. In both cases, the beam undergoes three further reflections (VDM, V-KB, and $\mathrm{H}-\mathrm{KB}$ ), with the first two being in the vertical plane. In total, the FEL beam experiences six reflections for DiProI (four horizontally deflecting and two vertically deflecting) and seven for LDM (five horizontally and two vertically). All mirrors are planar except the two KB refocusing elements (see below). It is possible to control the focus of the beam by adjusting the focal spot position and dimension to the requirements of the running experiment. This task is accomplished with an active-optics system, installed on both DiProI and LDM. Each system consists of a pair of 10-mm-thick mirrors arranged in a KB configuration; the mirrors are plane in their relaxed configuration and can be mechanically bent to the desired curvature [51].

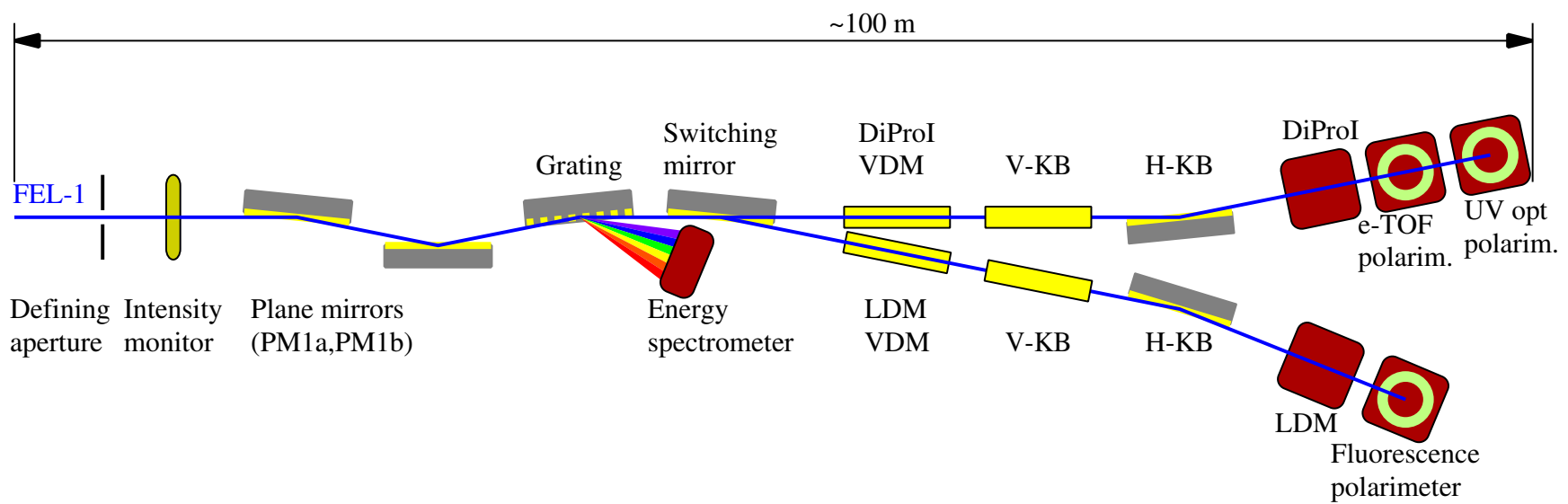

FIG. 2. Schematic layout of the PADReS systems with the three installed polarimeters: a VUV optical polarimeter and an electron time-of-flight (e-TOF) polarimeter have been installed in the DiProI beam line, and a fluorescence polarimeter has been installed in the LDM beam line. 
TABLE IV. Main optical parameters and physical description of the mirrors and grating of PADReS.

\begin{tabular}{|c|c|c|c|c|c|c|c|}
\hline \multirow[b]{2}{*}{ Name } & \multirow[b]{2}{*}{ Grazing angle $\left({ }^{\circ}\right)$} & \multicolumn{2}{|c|}{ Coating } & \multirow[b]{2}{*}{ Substrate } & \multicolumn{2}{|c|}{ Optical area } & \multirow[b]{2}{*}{ Deflection } \\
\hline & & Material & Thickness (nm) & & $\overline{\text { Length (mm) }}$ & $\overline{\text { Width (mm) }}$ & \\
\hline PM1a & 2.5 & $\mathrm{C}$ & 50 & $\mathrm{Si}$ & 250 & 20 & Horizontal \\
\hline PM1b & 5 & $\mathrm{C}$ & 50 & $\mathrm{Si}$ & 250 & 20 & Horizontal \\
\hline LE grating ${ }^{\mathrm{a}}$ & 2.5 & $\mathrm{C}$ & 50 & Fused silica & 250 & 25 & Horizontal \\
\hline LDM switching & 2 & $\mathrm{C}$ & 50 & $\mathrm{Si}$ & 480 & 20 & Horizontal \\
\hline LDM VDM & 2 & $\mathrm{Au}$ & 50 & $\mathrm{Si}$ & 390 & 16 & Vertical \\
\hline LDM V-KB & 2 & $\mathrm{Au}$ & 50 & Fused silica & 360 & 20 & Vertical \\
\hline LDM H-KB & 2 & $\mathrm{Au}$ & 50 & Fused silica & 360 & 20 & Horizontal \\
\hline DiProI VDM & 2 & $\mathrm{Au}$ & 50 & $\mathrm{Si}$ & 390 & 16 & Vertical \\
\hline DiProI V-KB & 2 & $\mathrm{Au}$ & 50 & Fused silica & 360 & 20 & Vertical \\
\hline DiProI H-KB & 2 & $\mathrm{Au}$ & 50 & Fused silica & 360 & 20 & Horizontal \\
\hline
\end{tabular}

${ }^{\mathrm{a}}$ Only the central $60 \mathrm{~mm}$ of this optic are ruled as a grating.

The e-TOF and the VUV-optical polarimeters were mounted in series downstream of the DiProI end station. The focal position of the radiation was moved downstream with respect to the nominal position so as to work with a more collimated beam. In this way, a spot size of $\approx 100 \times 100 \mu \mathrm{m}^{2}$ was obtained in the interaction volume of the e-TOF polarimeter. This configuration concurrently satisfied the optical requirements of the VUV-optical polarimeter in terms of both beam size $\left(2.4 \times 2.8 \mathrm{~mm}^{2}\right.$ at the entrance) and angular divergence. In the same way, at the LDM end station $\mathrm{a} \approx 1 \times 1 \mathrm{~mm}^{2}$ spot was obtained $\approx 1 \mathrm{~m}$ downstream of the standard (tight focus) conditions. In all cases, the optical alignment was checked first by means of Ce:YAG screens positioned along the photon beam and further refined using the signals obtained from the experimental apparatus.

As the optical system entails reflections in both horizontal and vertical planes, the beam transport can modify the final polarization of the incoming FEL pulses. The grazing incidence mirrors can have different reflection coefficients for $s$ and $p$ polarizations, resulting in a different transmission of the beam line for horizontal and vertical polarization.

Total transmission for the whole optical transport system from the undulator exit to DiProI and LDM was estimated by using the optical transport code IMD [53], together with
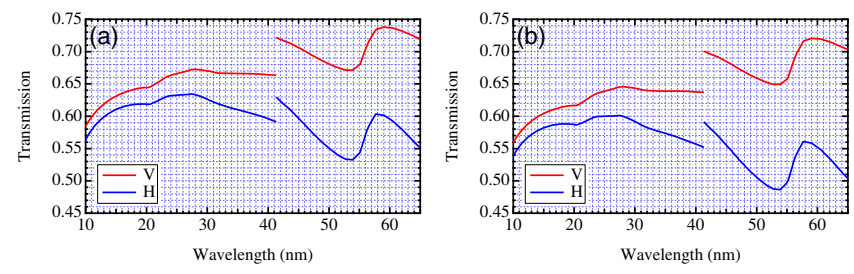

FIG. 3. Simulated transmission for horizontal and vertical polarization as a function of wavelength for the optical transport system to the DiProI (a) and LDM (b) end stations. The step at $\approx 41.3 \mathrm{~nm}(30 \mathrm{eV})$ is an artifact due to the use of two different data bases [54]. the mirror specifications reported in Table IV. A roughness equal to $0.5 \mathrm{~nm} \mathrm{rms}$ (representing the maximum rms roughness actually measured on one of the optics, the others having smaller roughness values) and an estimated carbon contamination of $20 \mathrm{~nm}$ were taken into account. Results of these simulations are reported in Fig. 3.

Since there are more reflections in the horizontal than in the vertical plane, the transmission for horizontally polarized radiation is, as expected, less efficient than for vertically polarized radiation. This difference, almost negligible around $10 \mathrm{~nm}$, becomes more important at longer wavelengths. The net horizontal transmission to LDM is slightly less than that to DiProI because of the additional horizontal reflection.

Besides affecting the transmission, the transport optics may also contribute a different phase delay for the horizontal and vertical polarization components. While this effect can be completely neglected for incoming radiation with perfectly horizontal or vertical polarization, it has to be seriously taken into account when the polarization of the FEL light is circular or elliptical.

The phase delay for horizontal and vertical polarization has been estimated using Fresnel equations and the refraction index for the PADReS mirror coatings as reported in Ref. [54]. The predicted phase delay between horizontal and vertical polarization as a function of the wavelength is shown in Fig. 4 for the DiProI and LDM beam lines. In this case too, the estimation has been
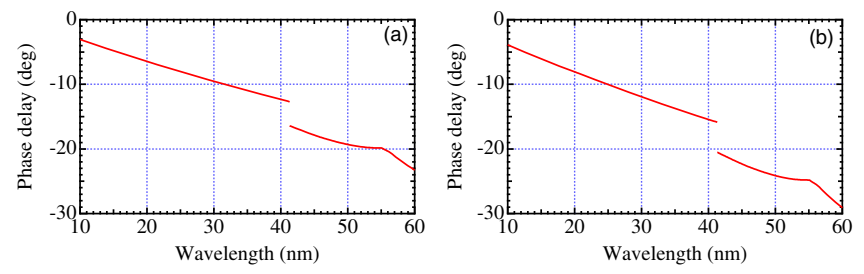

FIG. 4. Simulated phase delay between horizontal and vertical polarization versus wavelength for the optical transport system to DiProI (a) and LDM (b). See Fig. 3 for the step at $\approx 41.3 \mathrm{~nm}$. 
obtained by taking into account optics roughness and contamination.

\section{B. The VUV-optical polarimeter}

The VUV-optical polarimeter is an all-optical device based on the well-known polarimetry scheme [22,55] that uses a polarizer and an analyzer, which are rotated independently around the optical axis, to retrieve the complete state of polarization of an electromagnetic wave. At infrared or visible wavelengths, this type of polarimetry measurement can be done by using transparent optics, i.e., in transmission as indeed done with the fluorescence polarimeter. However, the extension to the vacuumultraviolet or soft x-ray spectral range requires the use of reflective optics since highly transparent materials are not available at such short wavelengths. At each reflection onto an optical element of the polarimeter, the $s$ and $p$ components of the electric field experience different reflectivities, respectively $R_{s}$ and $R_{p}$, and phase shifts, respectively $\varphi_{s}$ and $\varphi_{p}$. This difference enables the realization of an XUV linear (low $R_{p} / R_{s}$ ratio) or circular $\left(90^{\circ}\right.$ phase-shift difference) polarizer and analyzer.
Figure 5 is a sketch of the whole system. The polarizer consists of four identical fused-silica mirrors, coated with a 35-nm-thick layer of molybdenum capped with $5 \mathrm{~nm}$ of $\mathrm{B}_{4} \mathrm{C}$.

This particular coating is optimized for circularly polarized radiation in the 20-35-nm-wavelength range [8]. The polarizer has been fully characterized at the synchrotron light source BESSY II in Berlin. For the experiment carried out at FERMI, the four mirrors were set at a grazing angle of $20^{\circ}$ corresponding to a trade-off between the $R_{p} / R_{s}$ ratio, phase-shift difference, and overall transmission.

The analyzer is made of an uncoated fused-silica mirror set at an incidence angle of $45^{\circ}$. At this angle, the $R_{p} / R_{s}$ ratio for this mirror is very low in the wavelength range considered, making it a very efficient analyzer. The reflected signal is gathered by a $1 \times 1 \mathrm{~cm}^{2} \mathrm{XUV}$ photodiode.

The angles formed by the polarizer and by the analyzer with the horizontal plane are denoted in Fig. 5 as $\alpha$ and $\beta$, respectively. Using the Müller formalism [22,55], the intensity of the light arriving at the photodiode is then theoretically given by the following expression:

$$
\begin{aligned}
I_{\text {out }} \propto & S_{0}-S_{1} \cos 2 \psi_{1} \cos 2 \alpha S_{2} \cos 2 \psi_{1} \sin 2 \alpha+S_{0} \cos 2 \psi_{1} \cos 2 \psi_{2} \cos 2 \alpha \cos 2 \beta+S_{0} \cos 2 \psi_{1} \cos 2 \psi_{2} \sin 2 \alpha \sin 2 \beta \\
& +S_{3} \sin 2 \psi_{1} \cos 2 \psi_{2} \sin \phi_{1} \sin 2 \alpha \cos 2 \beta-S_{3} \sin 2 \psi_{1} \cos 2 \psi_{2} \sin \phi_{1} \cos 2 \alpha \sin 2 \beta \\
& -\frac{S_{1}}{2} \cos 2 \psi_{2} \cos 2 \beta\left(1+\sin 2 \psi_{1} \cos \phi_{1}\right)-\frac{S_{2}}{2} \cos 2 \psi_{2} \sin 2 \beta\left(1+\sin 2 \psi_{1} \cos \phi_{1}\right) \\
& -\frac{S_{1}}{2} \cos 2 \psi_{2} \cos 4 \alpha \cos 2 \beta\left(1-\sin 2 \psi_{1} \cos \phi_{1}\right)-\frac{S_{2}}{2} \cos 2 \psi_{2} \sin 4 \alpha \cos 2 \beta\left(1-\sin 2 \psi_{1} \cos \phi_{1}\right) \\
& +\frac{S_{2}}{2} \cos 2 \psi_{2} \cos 4 \alpha \sin 2 \beta\left(1-\sin 2 \psi_{1} \cos \phi_{1}\right)-\frac{S_{1}}{2} \cos 2 \psi_{2} \sin 4 \alpha \sin 2 \beta\left(1-\sin 2 \psi_{1} \cos \phi_{1}\right),
\end{aligned}
$$

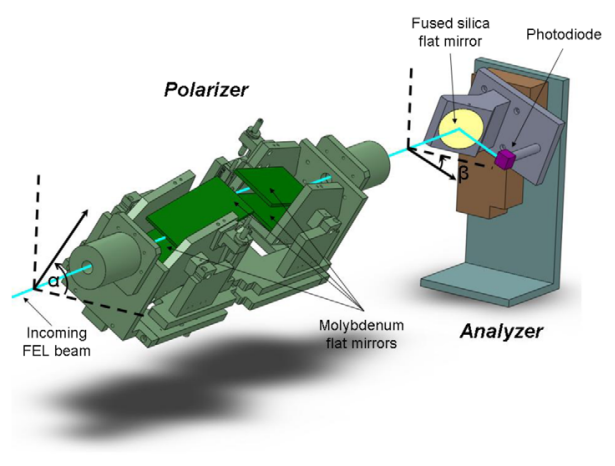

FIG. 5. VUV-optical polarimeter setup. The polarizer and the analyzer are placed into two successive vacuum chambers. The whole setup, approximately $1.5 \mathrm{~m}$ long, is positioned downstream of the DiProI end station. In front of and behind the polarizer, two irises of $5 \mathrm{~mm}$ diameter are used for aligning the polarimeter on the FEL axis. The $\alpha$ angle ranges from $-30^{\circ}$ to $+40^{\circ}$, the $\beta$ angle from $-170^{\circ}$ to $+170^{\circ}$. where $S_{0}, S_{1}, S_{2}$ and $S_{3}$ are the Stokes parameters [8] of the FEL light entering the polarimeter, $\psi=\tan \left(R_{p} / R_{s}\right)$ and $\phi=\varphi_{s}-\varphi_{p}$. Subscript 1 refers to the whole polarizer part (regrouping the four mirrors into a single composite optical element) and subscript 2 to the analyzer. Note that there is no $\phi_{2}$ component to be accounted for; i.e., the phase delay into the analyzer plays no role in the measurement. We recall that $S_{0}$ is a measure of the total intensity (polarized plus unpolarized components), while $S_{1}$ and $S_{2}$ characterize the linearly polarized part and $S_{3}$ the circularly polarized part.

\section{The e-TOF polarimeter}

The e-TOF polarimeter uses angle-resolved electron spectroscopy to determine the degree of linear polarization of the incident radiation. Comprised of 16 independent electron time-of-flight (e-TOF) spectrometers mounted in a plane perpendicular to the FEL beam (Fig. 6), the device 
can accurately determine the angular distribution of the photoelectrons that are emitted by the ionizing radiation. The angular distribution in this dipole plane depends on the degree and state of linear polarization, on the particular atomic gas target, and on which of its subshells is ionized [56]. By using an effusive gas beam to introduce different rare-gas atoms and measuring different subshells, we can unambiguously normalize the efficiency and transmission of the detectors and finally determine the degree, as well as the plane, of linear polarization. The absolute degree of circular polarization can be deduced if one assumes constant total polarization and determines the unpolarized background beforehand in the linear polarized mode of the light source.

The e-TOF polarimeter was designed for, and successfully tested at, the Variable Polarization XUV Beamline P04 of PETRA III [57], with emphasis on highly efficient polarization determination. For the current FEL experiment, this kind of polarimeter is complementary to the VUV and fluorescence optical polarimeters as it allows for discrete, single-shot polarimetry simultaneously with other experiments.

The data acquisition system (DAQ) of the e-TOF system was integrated into the FERMI-DAQ of the DiProI end station, and it tagged all acquired e-TOF data with the FERMI bunch number. This enabled us to do off-line crosscomparison of the polarimeter data with a set of corresponding FERMI beam parameters on a shot-by-shot basis. A detailed description of the setup will be reported in Ref. [58]. A brief description of the data-analysis procedure necessary for reconstructing the polarization state from the e-TOF spectrometer signals is given in Sec. IV B.

\section{The fluorescence polarimeter}

Polarization measurements at LDM were carried out by means of a novel experimental approach that takes advantage of the intrinsic polarization properties of fluorescence light from resonantly excited atoms. This method is based on the conversion of polarized VUV radiation to visible radiation with the same polarization parameters. The scheme, which is available whenever the decay pattern of the resonantly excited atom includes a suitable intermediate radiative step, is also supported by theoretical predictions [59]. A similar scheme was used in a previous experiment relying on synchrotron radiation [60]. With respect to direct polarization measurements at VUV wavelengths, this method has the significant advantage that polarization analysis of visible light is straightforward at the stage of data acquisition. Proper phase retarders and linearly polarizing analyzers based on transmission optics are also readily available, with ideal polarizing parameters. Exact quarter-wave phase retardation with no absorption (or isotropic absorption) and a high extinction ratio $\left(<10^{-3}\right)$ for the linear polarizer are the two key factors that allow a significantly simplified experimental setup,

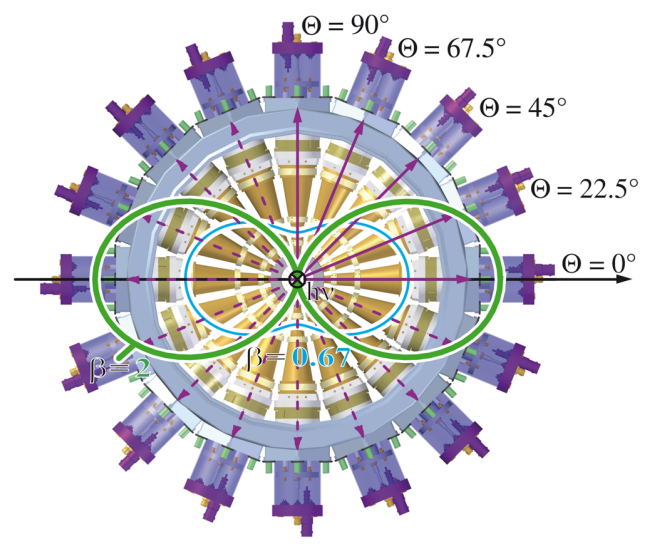

FIG. 6. Geometry of the 16-fold electron TOF spectrometer setup. The FEL beam enters perpendicular into the plane of the figure. The angular distribution for $\mathrm{Ne} 2 \mathrm{p}$ (blue) and $\mathrm{He} 1 \mathrm{~s}$ (green) photoelectrons is depicted for a wavelength of $26 \mathrm{~nm}$ and horizontally polarized light.

data-acquisition procedure, and, most importantly, dataanalysis procedure.

The polarization measurements presented here are based on resonance fluorescence from atomic helium. The chosen excitation and decay pattern is as follows: Absorption of VUV radiation of $53.703 \mathrm{~nm}(23.087 \mathrm{eV})$ resonantly excites the ground state of the He atom to the $3{ }^{1} \mathrm{P}$ singlet state, corresponding to the atomic configuration $\mathrm{He}(1 \mathrm{~s} 3 \mathrm{p})$ [61]. This state can decay into two radiative channels: $98 \%$ back to the ground state and $2 \%$ to the $2{ }^{1} \mathrm{~S}$ state, whose configuration is $\mathrm{He}(1 \mathrm{~s} 2 \mathrm{~s})$, with emission of $501.5 \mathrm{~nm}(2.471 \mathrm{eV})$ photons, that is, green radiation [62]. Fluorescence decay of the latter transition preserves the polarization state of the primary beam when observed along the propagation direction. A detailed analysis of the process and of the physics involved is reported in Ref. [63].

The experimental apparatus used for this experiment was tested on a HHG source [64] prior to installation on the LDM beam line, and it is described in Fig. 7. A constant flow of He was injected at the interaction region from a needle type of inlet. The nominal pressure of the He flow

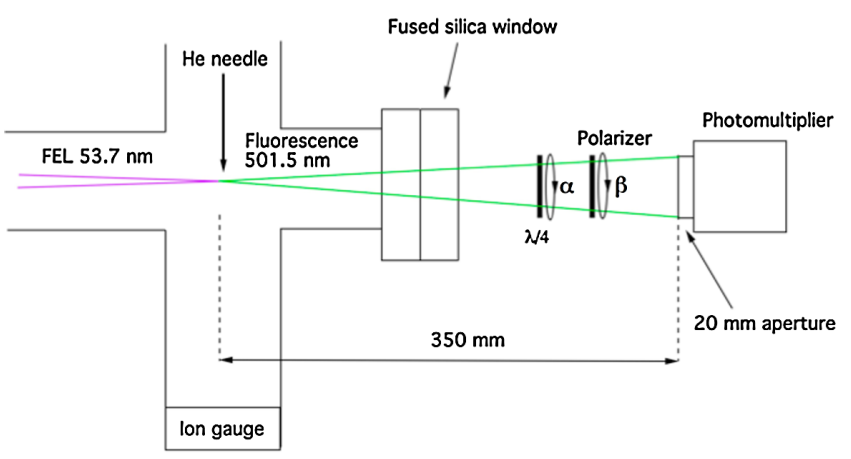

FIG. 7. Schematic diagram of the experimental apparatus for the polarization measurements at LDM. 
was $1 \times 10^{-5}$ mbar. The whole apparatus for the polarization measurements of the visible fluorescence was mounted in air and shielded from environmental visible light. This apparatus consists of a broadband $\lambda / 4$ phase retarder (ranging from 400 to $700 \mathrm{~nm}$ ) and an analyzer, both working in transmission. The rotation angle of the polarizing optics could be determined with an accuracy of $0.1^{\circ}$

The intensity of the fluorescence transmitted by the polarimeter was measured by means of a photomultiplier with a $20-\mathrm{mm}$-diameter aperture. The distance between the interaction region and the detector was $350 \mathrm{~mm}$, giving a total angular acceptance of about $3^{\circ}$. Polarization analysis was carried out by monitoring the intensity as a function of the rotation angle of each of the two polarizing optics. Vacuum and air were separated by a 3-mm-thick fused silica window, DUV grade, which has a transmission $>90 \%$ at a 501.5 -nm wavelength.

This window also acted as a beam stopper for the FEL UV radiation. The 268.5-nm radiation from the FEL seed laser was filtered by means of an $\mathrm{Al}$ foil $(200 \mathrm{~nm}$ thick) inserted several meters upstream of the interaction region.
To minimize the probability of nonlinear processes, the FEL beam spot size at the interaction region was purposefully set to about $1 \mathrm{~mm}$ diameter (full width half maximum); this size is rather large when compared to typical experiments where focusing is in the range of a few tens of $\mu \mathrm{m}$. The spot-size adjustment was done by means of the adaptive $\mathrm{KB}$ mirrors.

The experimental method and theoretical formalism for determining the Stokes parameters of the visible fluorescence light from the analysis of the intensity transmitted by the polarizing optics are the same as those used for the UV multiple reflection optics presented in Sec. III B. Equation (1) again applies, once reflection coefficients are replaced by the appropriate transmission ones (the full theory of polarization analysis by means of reflection devices can be found in Refs. $[65,66])$.

The total intensity of the light transmitted by the polarimeter (retarder + analyzer) is derived from the input Stokes vector $S^{\text {in }}$ and the complex transmission coefficients of the polarimeter $[65,66]$. In the configuration used here, with a $\lambda / 4$ phase retarder and a high-extinction-ratio polarimeter, the equation for the transmitted light as measured by the detector $I_{d}$ can be simplified as

$$
I_{d}=K\left\{1+\frac{1}{2} S_{1}[\cos 2 \beta+\cos (4 \alpha-2 \beta)]+\frac{1}{2} S_{2}[\sin 2 \beta+\sin (4 \alpha-2 \beta)]+S_{3} \sin (2 \alpha-2 \beta)\right\}
$$

In Eq. (2), $K$ is a calibration factor, which accounts for the number of photons per pulse $\left(S_{0}\right)$, the fluorescence yield, the fine wavelength tuning of the FEL, the transmission through the optics, and the efficiency of the detector; $S_{1}, S_{2}, S_{3}$ are the normalized Stokes parameters of the incoming FEL radiation.

By measuring $I_{d}$ for different combinations of $\alpha$ and $\beta$, one can completely characterize the polarization state of the light. Consistently with Eq. (1), both $\alpha$ and $\beta$ are measured relative to the horizontal axis. The horizontal direction was determined by means of a mechanical level that defined the alignment of the breadboard supporting the polarimeter optics; this procedure yields an accuracy of about $1^{\circ}$. The choice of mechanically mounting the polarimeter parts on the breadboard allows us to maintain this alignment to an even higher level of accuracy.

\section{RESULTS}

The FEL radiation polarization properties were measured with the three setups described in the previous sections. While the VUV-optical and e-TOF systems could operate in parallel and thus simultaneously characterize the same discrete FEL pulses, the LDM fluorescence apparatus was operated as an independent experiment. It was physically installed on a different branch line of PADReS, and, as mentioned, required a different setup of the FEL, in order for the output photon energy to be matched to the desired atomic resonance.

\section{A. VUV-optical measurements and analysis}

For each FEL configuration characterized by the optical polarimeter, the $\beta$ angle was scanned for various $\alpha$ angles (see Fig. 5). A typical measurement of the intensity collected by the photodiode at a fixed $\alpha$ value is shown in Fig. 8. For each FEL shot, the signal on the photodiode is normalized to the intensity read using the PADReS gas-ionization-monitor diagnostic in order to account for the FEL shot-to-shot intensity fluctuations. The data analysis is performed on the points obtained by averaging the various measurements for each $\beta$ value. A least-squares fitting to Eq. (1) is then performed, allowing the retrieval of the Stokes parameters.

For each FEL configuration, the $\beta$ scans were repeated for various $\alpha$ values in order to improve the fitting procedure and to reduce the errors. While $\beta$ was scanned from $-170^{\circ}$ to $170^{\circ}$ with a $10^{\circ}$ step, typical $\alpha$ values used for the scans were $-30^{\circ},-22^{\circ},-15^{\circ},-7^{\circ}, 0^{\circ}, 7^{\circ}, 15^{\circ}, 22^{\circ}$, $30^{\circ}$, and $40^{\circ}$.

The acquired data have been analyzed with different fitting algorithms, and the results are in good agreement. The accuracy in the absolute values for the determined 


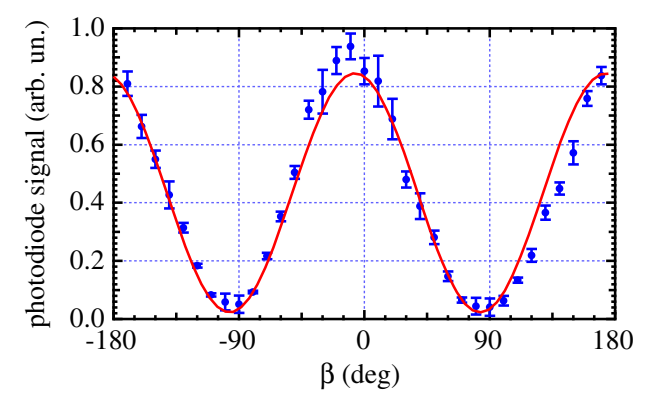

FIG. 8. Typical data set of a $\beta$ scan acquired with the optical polarimeter. The normalized detector signal averaged over 50 FEL shots (blue points), and the rms errors for each $\beta$ value are plotted versus the polarizer angle $\beta$. In red, the best fit is also shown. Reported data refer to the 26-nm vertical polarization with $\alpha=7^{\circ}$.

Stokes parameters has been estimated to be better than 0.05 .

Figure 9 reports a collection of the measured average signals for various $\alpha$ and $\beta$, together with the fitted curves. Data reported in Figs. 8 and 9 refer to the FEL optimized at $26 \mathrm{~nm}$ for vertical polarization. The fitting algorithm applied to this data set defines the Stokes parameters $S=[1,-0.97,0.03,0.07]$ that characterize the measured
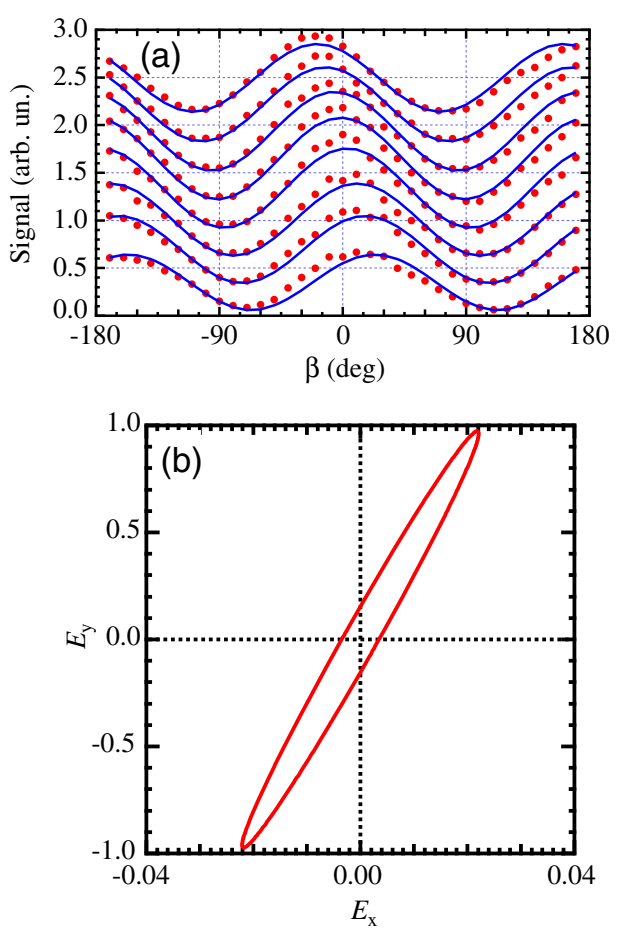

FIG. 9. (a) Complete data set of the scans for 26-nm vertical polarization. Red points report the average signal for each value of $\alpha$ and $\beta$. Blue lines show the fitted curves obtained from the various $\beta$ scans, allowing the retrieval of the FEL Stokes parameters. (b) Polarization ellipse reconstructed with the Stokes parameters obtained from the fit. Note the expanded horizontal scale.
TABLE V. Results of the analysis of the VUV-optical polarimeter data. Total degree of polarization and Stokes parameters, normalized to $S_{0}$, are reported for different undulator settings and wavelengths.

\begin{tabular}{llcccc}
\hline \hline $\begin{array}{l}\text { Wavelength } \\
(\mathrm{nm})\end{array}$ & $\begin{array}{l}\text { Nominal FEL } \\
\text { polarization }\end{array}$ & $\begin{array}{c}\text { Measured } \\
\text { polarization }\end{array}$ & $S_{1} / S_{0}$ & $S_{2} / S_{0}$ & $S_{3} / S_{0}$ \\
\hline 26 & Linear vertical & 0.97 & -0.97 & 0.03 & 0.07 \\
26 & Circular right & 0.96 & -0.02 & 0.05 & 0.96 \\
32 & Linear vertical & 0.96 & -0.96 & 0.02 & 0.06 \\
32 & Circular left & 0.93 & -0.05 & -0.19 & -0.91 \\
\hline \hline
\end{tabular}

FEL radiation. As is evident from Fig. 9(a), the polarization state found returns a consistently good fit for all the scans done at different values of $\alpha$. Figure 9(b) shows the reconstructed polarization ellipse obtained from the measured Stokes parameters, corresponding to a degree of polarization for the FEL of $P=0.97$.

The same analysis procedure has been followed for all the data sets acquired in different polarization states and wavelengths, with final results summarized in Table V. Data corresponding to horizontal polarization could not be characterized because of the low photon flux reaching the photodiode in this condition, caused by the low reflectivity of the polarizer for this polarization. In every case, the total degree of polarization is above 0.9 and the retrieved Stokes parameters match the nominal polarization settings of the undulators well. One should note that for the circular cases, non-negligible values of $S_{1}$ and $S_{2}$ are obtained. These values nevertheless are consistent with the expected effect of the beam-line optics upon the polarization of the FEL light (see Figs. 3 and 4), as will be discussed at the end of Sec. IV C.

Furthermore, in addition to the Stokes parameters, the data fitting allows retrieval of the $\psi_{1}, \psi_{2}$, and $\phi_{1}$ parameters [see Eq. (1)], i.e., the $R_{p} / R_{s}$ ratio in the polarizer and in the analyzer, and the phase delay in the polarizer. The values that have been found are, respectively, $0.50,0.14$, and $78.0^{\circ}$ at $26-\mathrm{nm}$ radiation wavelength and $0.43,0.20$, and $78.3^{\circ}$ at $32 \mathrm{~nm}$. The phase delay and the $R_{p} / R_{s}$ ratio in the polarizer are in agreement with previous calibration; this is a further check of the quality of the data analysis.

\section{B. e-TOF polarimeter}

After calibrating the 16-fold angle-resolving photoelectron spectrometer setup that is described in a forthcoming paper [58], the analysis of the TOF signals acquired by the polarimeter allowed reconstruction of the angular distribution of the photoelectrons ionized by the polarized FEL pulses (Fig. 10). For each individual FEL shot, the 16 signals giving the probability distribution function of the electron can be fit by a theoretical prediction that takes into account the physics of the ionization process. In the dipole approximation, the electron angular distribution can be described by the following expression: 
TABLE VI. Summary of the measured average degree of polarization for various FEL configurations. $S_{1}$ and $S_{2}$ are extracted from $P_{\text {lin }}$ through trigonometry, and $S_{3}$ is calculated assuming perfectly polarized light; the measurement setup cannot distinguish between unpolarized and circularly polarized light.

\begin{tabular}{|c|c|c|c|c|c|c|}
\hline Wavelength (nm) & FEL polarization & $P_{\text {lin }}$ & $\psi\left(\left(^{\circ}\right)\right.$ & $S_{1} / S_{0}$ & $S_{2} / S_{0}$ & $S_{3} / S_{0}$ \\
\hline 26 & Linear vertical & $0.97 \pm 0.02$ & $89.7 \pm 1$ & -0.97 & 0.01 & 0.25 \\
\hline 26 & Linear horizontal & $0.94 \pm 0.02$ & $0.4 \pm 1$ & 0.94 & 0.01 & 0.34 \\
\hline 26 & Circular left & $0.11 \pm 0.02$ & $50 \pm 6$ & -0.02 & 0.11 & -0.99 \\
\hline 26 & Circular right & $0.11 \pm 0.02$ & $127 \pm 6$ & -0.03 & -0.10 & 0.99 \\
\hline 32 & Linear vertical & $0.90 \pm 0.02$ & $91.3 \pm 1$ & -0.90 & -0.04 & 0.43 \\
\hline 32 & Linear horizontal & $0.97 \pm 0.02$ & $-1.2 \pm 1$ & 0.97 & -0.04 & 0.23 \\
\hline 32 & Circular left & $0.10 \pm 0.02$ & $124 \pm 5$ & -0.04 & -0.09 & -0.99 \\
\hline 32 & Circular right & $0.14 \pm 0.02$ & $53 \pm 6$ & -0.04 & 0.13 & 0.99 \\
\hline
\end{tabular}

$$
P(\theta)=1+\frac{\beta^{\mathrm{TOF}}}{4}\left\{1+3 P_{\text {lin }} \cos [2(\theta-\psi)]\right\}
$$

Here, $P_{\text {lin }}$ is the degree of linear polarization $\left(P_{\text {lin }}=\sqrt{S_{1}^{2}+S_{2}^{2}}\right), \psi$ is the direction of the linear polarization, and $\beta^{\text {TOF }}$ describes the angular anisotropy parameter. For the case of $\mathrm{He}$ atoms, $\beta^{\mathrm{TOF}}=2$. Please note that this $\beta^{\mathrm{TOF}}$ parameter is not an angle, nor it is related to the parameter $\beta$ appearing in Eqs. (1) and (2).

As already anticipated, the e-TOF polarimeter in the configuration used here can only directly measure the degree of linear polarization $\left(P_{\text {lin }}\right)$. The degree of circular polarization $\left(P_{\text {circ }}\right)$, without distinction between left and right polarizations, can only be estimated by the formula $P_{\text {circ }}=\sqrt{1-P_{\text {lin }}^{2}}$ under the assumption that the radiation is fully polarized. This assumption is reasonable for the case of the FERMI FEL since it has been demonstrated both by measurement of the linear polarization and by measurements done with the other two polarimeters that the degree of polarization is very high.

Measurements were taken with the e-TOF setup at various wavelengths and polarizations. Table VI summarizes the results of the measurements done for the four polarizations at $26-\mathrm{nm}$ and $32-\mathrm{nm}$ wavelengths.

\section{Polarization sensitivity to FEL settings}

The capability for fast, single-shot polarization measurements provided by the e-TOF polarimeter has been exploited to study the sensitivity of FEL polarization to the various electron-beam and seed-laser parameters typically used to optimize the FEL output radiation. These parameter studies were made primarily with the FEL operating at a 32-nm wavelength in horizontal polarization. Several series of polarization measurements were conducted as the seeding (e.g., power, relative timing), and FEL parameters (e.g., dispersion-section strength, laserheater power, number of radiators on resonance) were changed systematically.

A representative data set of this kind of study is shown in Fig. 11. In this particular case, the seed-laser power has been scanned from the minimum value that is necessary for producing a coherent signal up to very high values that far exceed the optimum and generate a strong overbunching, as is evident from structures appearing in the FEL output spectrum (notably characterized by a double peak [67]). During this scan, we simultaneously acquired the FEL spectrum [Fig. 11(a)], the FEL intensity as measured by the PADReS intensity monitor [Fig. 11(c)], and the 16 e-TOF signals [Fig. 11(b)]. These signals could be directly correlated since all acquisitions were tagged with the bunch number.

The results summarized in Fig. 11 provide strong evidence that the degree of polarization, as measured by the e-TOF, is insensitive to the seed power level and to its effect on the FEL output power and spectrum. Similarly,
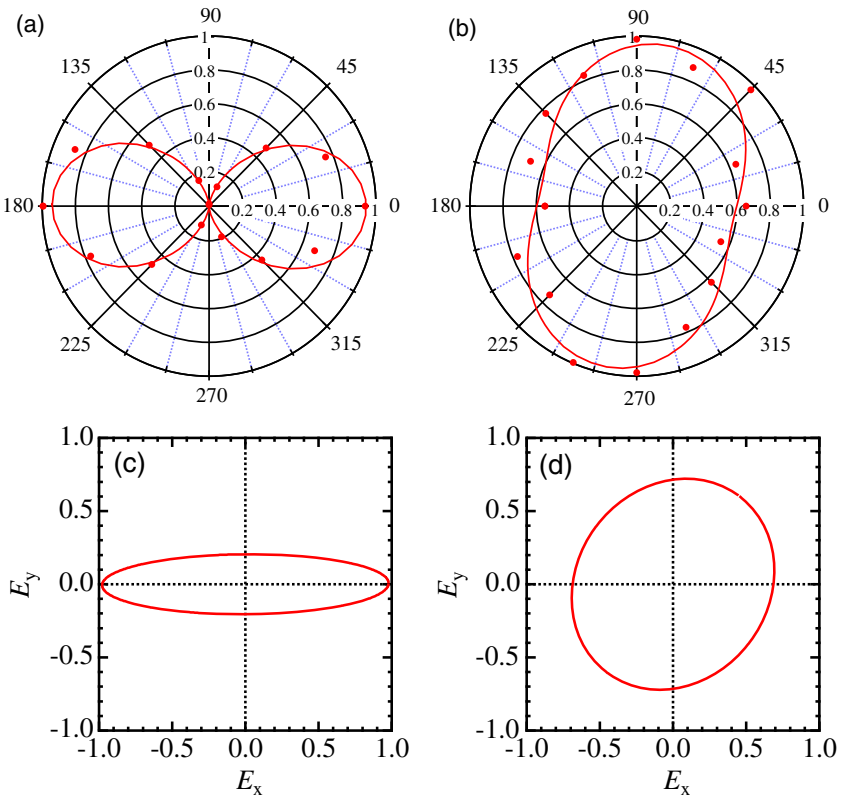

FIG. 10. Example of the processed signal of the e-TOF showing single shot data for the electron-beam distribution on He for the FEL at 32-nm horizontally polarized (a) and circularly right polarized (b). Panels (c) and (d) report the corresponding reconstructed ellipses. 

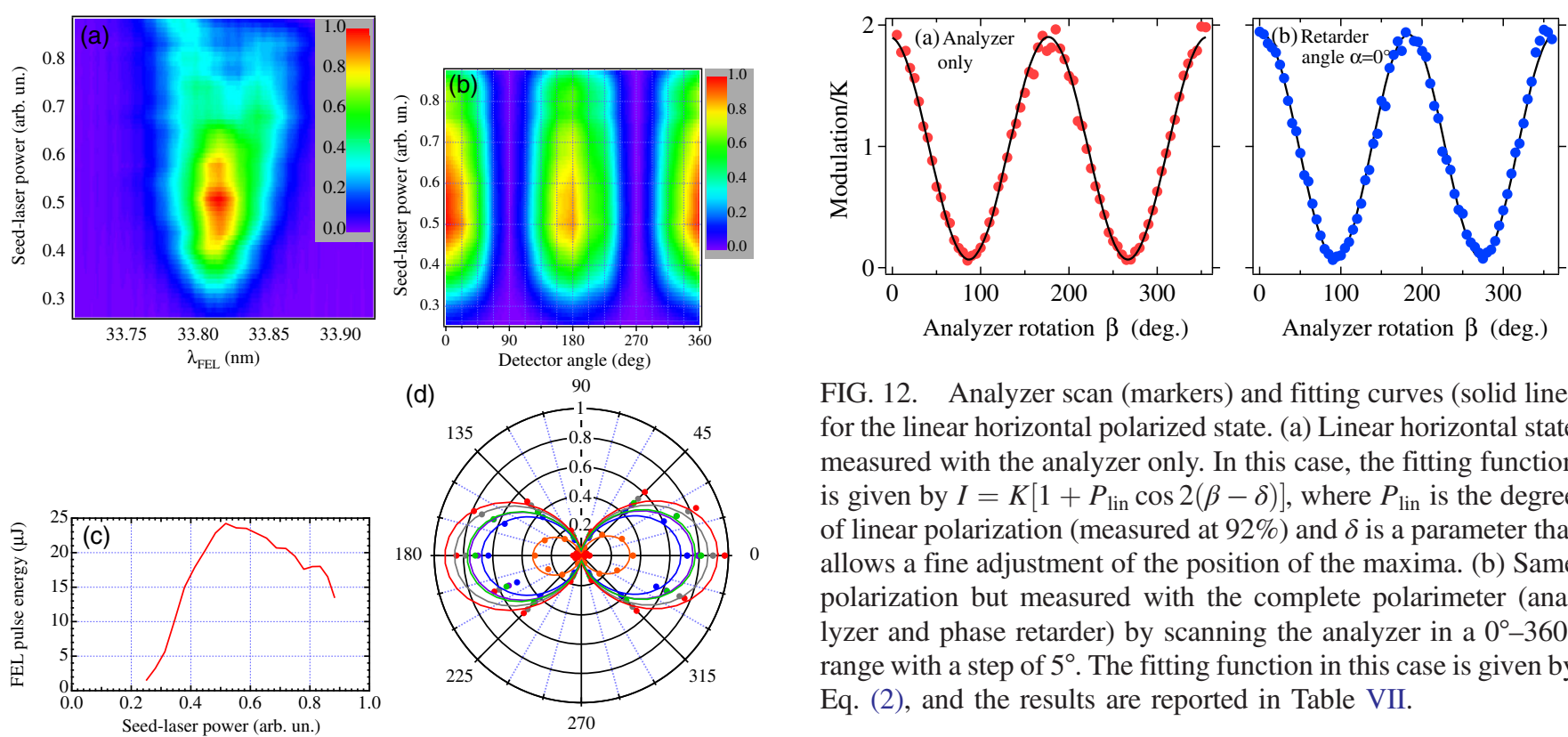

FIG. 12. Analyzer scan (markers) and fitting curves (solid line) for the linear horizontal polarized state. (a) Linear horizontal state measured with the analyzer only. In this case, the fitting function is given by $I=K\left[1+P_{\text {lin }} \cos 2(\beta-\delta)\right]$, where $P_{\text {lin }}$ is the degree of linear polarization (measured at $92 \%$ ) and $\delta$ is a parameter that allows a fine adjustment of the position of the maxima. (b) Same polarization but measured with the complete polarimeter (analyzer and phase retarder) by scanning the analyzer in a $0^{\circ}-360^{\circ}$ range with a step of $5^{\circ}$. The fitting function in this case is given by Eq. (2), and the results are reported in Table VII.

FIG. 11. Evolution of FEL and polarization as a function of the seed-laser power: (a) FEL spectrum, (b) average signal measured by the 16 e-TOF, and (c) FEL energy per pulse as a function of the seed-laser power. Panel (d) reports the polar plot of 16 e-TOF average signals for a few seed-laser intensities and shows that the angular distribution of the signal over the 16 detectors is essentially maintained at all seeding intensities.

the degree of polarization remained insensitive and uncorrelated to other external seed, electron-beam, and undulator parameters (other than undulator polarization, as reported in Table VI). Changes of critical parameters, such as the seed-laser timing, undulator resonance, and strength of the dispersive section to the extent that the FEL intensity is suppressed by more than a factor 2 and the spectrum is significantly affected, have not shown any effect on the degree of the measured polarization. It is important to point out that normal shot-to-shot fluctuations of machine parameters induce variations of FEL intensity and other spectral properties that are much smaller $[33,34]$ than those intentionally induced in the mentioned parameter scans. Indeed, preliminary analysis does not show any correlation between the degree of polarization and machine parameters.

\section{Fluorescence polarimeter}

Polarization measurements at LDM were carried out for linear horizontal (Fig. 12), circular right (data not shown), and circular left (Fig. 13) states for the FEL operated at a wavelength of $53.7 \mathrm{~nm}$. The curves shown in these graphics were normalized to the experimental amplitude parameter $k I_{0}$ that was determined via the fitting procedure.

All analyzer scans were normalized to the FEL energyper-pulse measured with the PADReS gas ionization monitor. After subtraction of the background fluorescence

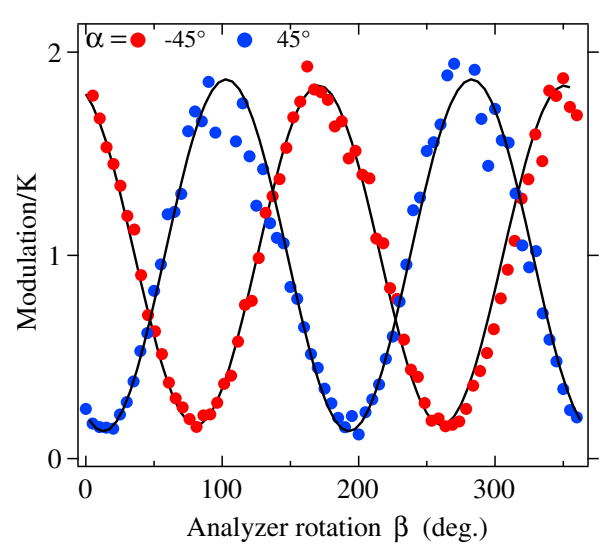

FIG. 13. Analyzer scans for the state of nominal circular-left polarization at $\alpha=-45^{\circ}$ (red) and $\alpha=-45^{\circ}$ (blue). These data allow us to measure $S_{2}$ and $S_{3}$.

(emitted by the fused silica window), the Stokes parameters were determined by means of a fit to the function given in Eq. (2). The results of the fitting procedure for the three polarization states are shown in Table VII.

Horizontal linear polarization was measured both with and without a phase retarder; the two measurements provide the same degree of polarization $(P=0.92)$. This result shows that the polarization is purely linear. The maxima (minima) appear at the same angular position for the two curves; this is an indication of the good internal alignment of the polarimeter.

However, these maxima do not appear exactly at $0^{\circ}$; they exhibit a shift of $\approx 3^{\circ}$ that results in a nonzero $S_{2}$. In principle, one cannot rule out a systematic error introduced by a rotation of the polarimeter in the laboratory reference frame, even though $3^{\circ}$ is large compared to the accuracy of the initial positioning. 
TABLE VII. Best-fit parameters for different FEL nominal polarization states (see text for the fitting procedure).

\begin{tabular}{lcrrr}
\hline \hline $\begin{array}{l}\text { Nominal FEL } \\
\text { polarization }\end{array}$ & $\begin{array}{c}\text { Measured } \\
\text { polarization }\end{array}$ & $S_{1} / S_{0}$ & $S_{2} / S_{0}$ & $S_{3} / S_{0}$ \\
\hline Linear horizontal & 0.92 & 0.92 & 0.11 & 0.00 \\
Circular right & 0.92 & -0.07 & 0.21 & 0.89 \\
Circular left & 0.93 & -0.20 & -0.31 & -0.85 \\
\hline \hline
\end{tabular}

In the case of circular-left polarization, the $\beta$ scan of the analyzer has been performed by setting the polarizer angle $\alpha$ at $0^{\circ}$ and $\pm 45^{\circ}$. Table VII summarizes the Stokes parameters obtained for all the polarization cases studied with the LDM polarimeter.

A remarkable result pertaining to the circular polarization is the substantial amounts of $S_{1}$ and $S_{2}$ measured. These parameters, expected to be strictly zero for a perfectly circularly polarized light, definitely point to elliptical polarization states. Given the relatively long wavelength used in this experiment, the role of the photon-transport system as a potential polarizer source needs to be considered carefully. Indeed, predictions anticipated in Fig. 4 suggest that a phase delay of more than 10 degrees may occur between the horizontal and vertical polarization components. The measured tilt of the polarization ellipse is compatible with this simulated phase delay for the beam-line optics.

\section{Summary}

The results of polarization measurements obtained with the three polarimeters summarized in Tables V, VI, and VII indicate a high degree of polarization for all the polarizations and wavelengths studied. The tilted elliptical

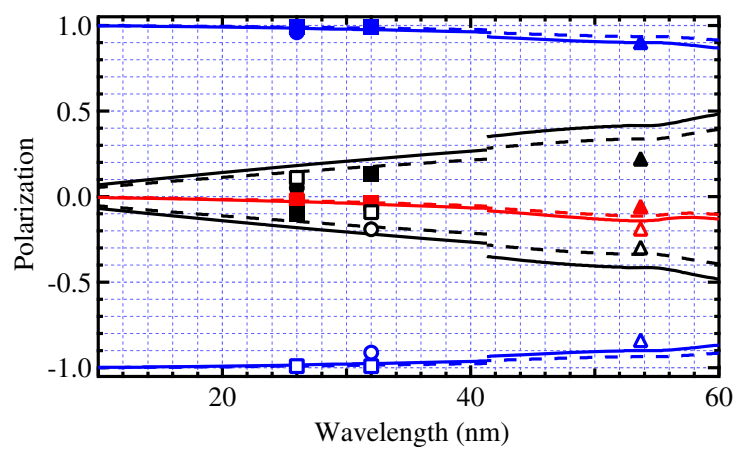

FIG. 14. Predicted impact of the LDM (dashed lines) and DiProI (continuous line) beam-line optics on the Stokes parameters $\left(\right.$ red $=S_{1} / S_{0}$, black $=S_{2} / S_{0}$, blue $=S_{3}$ ) for a circular polarized light produced at the undulator. Predictions are shown together with measured Stokes parameters at the experimental stations (circular-right filled symbols and circular-left empty symbols) reported in Tables V, VI, and VII and obtained with the LDM setup (triangles), VUV optical polarimeter (circles), and e-TOF polarimeter (squares). See Fig. 3 for the step at $\approx 41.3 \mathrm{~nm}$. polarization measured for circularly polarized undulators is explained as an effect of the beam-line transport optics and is indeed more prominent for longer wavelength, where the beam-line polarizing effect is stronger. The small amount of nonpolarized light measured can be attributed to the beam-line optics with residual roughness and diffraction effects; in a more refined analysis, the contribution of off-axis emission should be considered too.

In Fig. 14, the measured Stokes parameters in the case of circularly polarized FEL light are reported, together with the predicted beam-line-induced polarizing effect (including roughness and contamination) for both the LDM and DiProI beam-line optics. The figure shows that the measured behavior is in very good agreement with the optical calculations accounting for the different response of the mirror to $s$ and $p$ polarization components.

\section{CONCLUSIONS}

We have conducted a detailed study of the FEL polarization generated by FERMI FEL-1 in the VUV soft x-ray spectral range over various different wavelengths and machine conditions. Three independent measurement devices and methods have been implemented, and the obtained results have been cross-checked for mutual consistency.

Our results indicate that the polarization properties of the high-power coherent light from FERMI FEL-1 are those expected and that the polarization state may be finely controlled and adjusted by changing the undulator polarization according to the needs of the user experiments within less than one minute. The three independent methods have measured polarization degrees greater than 0.9 for all linear and circular polarization states over the wavelength range $26-55 \mathrm{~nm}$. As expected, the degree of output polarization is insensitive to seed-laser and electronbeam parameters.

A small ellipticity has been measured in the case of circular undulator polarization. This ellipticity becomes more significant at longer wavelengths, where the dichroism of the grazing incidence mirrors, used to deliver the FEL light to the experimental stations, becomes larger. This behavior is in good agreement with optical calculations that take into account the effects of the dichroism of downstream transport mirrors. This agreement suggests that for those experiments requiring a particularly high degree of circular polarization, a proper tuning of the undulators could be used to compensate this beam-line dichroism, thus reducing the spurious $S_{1}$ component that is ultimately incident onto the experimental stations.

\section{ACKNOWLEDGMENTS}

We acknowledge the entire FERMI team for their contribution, which made possible these measurements with the continuous support in running the free electron laser. We thank M. Lonza, R. Borghes, and L. Pivetta for 
their support in the integration of the various instruments with the FERMI control system, and M. Kiskinova, Z. Huang, A. Lutman, and D. Garzella who contributed with many insightful discussions. The authors are indebted to $\mathrm{M}$. Svandrlik and F. Parmigiani for their advice and suggestions. M.C. and G. D. N. acknowledge support from the Italian-Slovenian Crossborder Cooperation Programme (CITIUS Project No. 2013/2010090600115298). The work of G.D. N., P.R.R., D. G., C. G., and B.R. is partially sponsored by the Slovenian Research Agency (Contract No. Q1-0015). M. I. acknowledges financial support from the Volkswagen Foundation within the Peter Paul EwaldFellowship. A. Kn. thanks A. Ehresmann for support and acknowledges the contribution of the State Initiative for the Development of Scientific and Economic Excellence (LOEWE) in the LOEWE-Focus ELCH. G. L., B. V., J. L., and P.Z. acknowledge financial support from the Triangle de la Physique, under the IMMAGE and POLIMMAGE contracts, for the realization of the mirror-based polarizer. G. L. and P.Z. acknowledge the ESF COST Action MP1203 for partial financial support. The resources that made the construction of FERMI possible were obtained and managed by G. Comelli, A. Franciosi, and C. Rizzuto. This work was funded by the FERMI project of Elettra-Sincrotrone Trieste, partially supported by the Ministry of University and Research (Grants No. FIRBRBAP045JF2 and No. FIRB-RBAP06AWK3).

[1] J. Cooper and R. N. Zare, Angular Distribution of Photoelectrons, J. Chem. Phys. 48, 942 (1968); K. L. Reid, Photoelectron Angular Distributions, Annu. Rev. Phys. Chem. 54, 397 (2003); N. M. Kabachnik, S. Fritzsche, A. N. Grum-Grzhimailo, M. Meyer, and K. Ueda, Coherence and Correlations in Photoinduced Auger and Fluorescence Cascades in Atoms, Phys. Rep. 451, 155 (2007).

[2] M. H. M. Janssen and I. Powis, Detecting Chirality in Molecules by Imaging Photoelectron Circular Dichroism, Phys. Chem. Chem. Phys. 16, 856 (2014).

[3] J.-Y. Bigot, M. Vomir, and E. Beaurepaire, Coherent Ultrafast Magnetism Induced by Femtosecond Laser Pulses, Nat. Phys. 5, 515 (2009).

[4] S. Eisebitt, J. Lüning, W. F. Schlotter, M. Lörgen, O. Hellwig, W. Eberhardt, and J. Stöhr, Lensless Imaging of Magnetic Nanostructures by X-ray Spectro-holography, Nature (London) 432, 885 (2004).

[5] C. Boeglin, E. Beaurepaire, V. Halté, V. López-Flores, C. Stamm, N. Pontius, H. A. Dürr, and J.-Y. Bigot, Distinguishing the Ultrafast Dynamics of Spin and Orbital Moments in Solids, Nature (London) 465, 458 (2010).

[6] J. Itatani, J. Levesque, D. Zeidler, H. Niikura, H. Pépin, J. C. Kieffer, P. B. Corkum, and D. M. Villeneuve, Tomographic Imaging of Molecular Orbitals, Nature (London) 432, 867 (2004).

[7] P. Eckle, M. Smolarski, P. Schlup, J. Biegert, A. Staudte, M. Schöffler, H. G. Muller, R. Dörner, and U. Keller, Attosecond Angular Streaking, Nat. Phys. 4, 565 (2008);
P. Eckle, A. N. Pfeiffer, C. Cirelli, A. Staudte, R. Dorner, H. G. Muller, M. Buttiker, and U. Keller, Attosecond Ionization and Tunneling Delay Time Measurements in Helium, Science 322, 1525 (2008); M. Weger, J. Maurer, A. Ludwig, L. Gallmann, and U. Keller, Transferring the Attoclock Technique to Velocity Map Imaging, Opt. Express 21, 21981 (2013).

[8] D. H. Goldstein, Polarized Light, 2nd ed. (CRC Press, New York, 2003).

[9] J. Bahrdt, W. Frentrup, A. Gaupp, M. Scheer, W. Gudat, G. Ingold, and S. Sasaki, Elliptically Polarizing Insertion Devices at BESSY II, Nucl. Instrum. Methods Phys. Res., Sect. A 467-468, 21 (2001).

[10] D. Desiderio, S. Difonzo, B. Dlviacco, W. Jark, J. Krempasky, R. Krempaska, F. Lama, M. Luce, H. C. Mertins, M. Placentini et al., The Elettra Circular Polarization Beamline and Electromagnetic Elliptical Wiggler Insertion Device, Synchrotron Radiat. News 12, 34 (1999).

[11] H. Wang, P. Bencok, P. Steadman, E. Longhi, J. Zhu, and Z. Wang, Complete Polarization Analysis of an APPLE II Undulator Using a Soft X-ray Polarimeter, J. Synchrotron Radiat. 19, 944 (2012).

[12] B. M. Kincaid, A Short-Period Helical Wiggler as an Improved Source of Synchrotron Radiation, J. Appl. Phys. 48, 2684 (1977).

[13] G. Dattoli and L. Bucci, Variably Polarizing Undulators: Spectral Properties and Free Electron Laser Gain, Opt. Commun. 177, 323 (2000).

[14] B. W. J. McNeil and N. R. Thompson, X-ray Free-Electron Lasers, Nat. Photonics 4, 814 (2010).

[15] W. Ackermann et al., Operation of a Free-Electron Laser from the Extreme Ultraviolet to the Water Window, Nat. Photonics 1, 336 (2007).

[16] P. Emma et al., First Lasing and Operation of an AngstromWavelength Free-Electron Laser, Nat. Photonics 4, 641 (2010).

[17] T. Ishikawa, H. Aoyagi, T. Asaka, Y. Asano, N. Azumi, T. Bizen, H. Ego, K. Fukami, T. Fukui, Y. Furukawa et al., A Compact X-ray Free-Electron Laser Emitting in the Sub-angstrom Region, Nat. Photonics 6, 540 (2012).

[18] S. Sasaki, Analyses for a Planar Variably-Polarizing Undulator, Nucl. Instrum. Methods Phys. Res., Sect. A 347, 83 (1994).

[19] A. B. Temnykh, Delta Undulator for Cornell Energy Recovery Linac, Phys. Rev. ST Accel. Beams 11, 120702 (2008).

[20] P. Rebernik Ribic and G Margaritondo, Status and Prospects of X-ray Free-Electron Lasers (X-FELs): A Simple Presentation, J. Phys. D 45, 213001 (2012).

[21] R. W. Schoenlein, S. Chattopadhyay, H. H. W. Chong, T. E. Glover, P. A. Heimann, C. V. Shank, A. A. Zholents, and M.S. Zolotorev, Generation of Femtosecond Pulses of Synchrotron Radiation, Science 287, 2237 (2000).

[22] B. Vodungbo, A. Barszczak Sardinha, J. Gautier, G. Lambert, C. Valentin, M. Lozano, G. Iaquaniello, F. Delmotte, S. Sebban, J. Lüning, and P. Zeitoun, Polarization Control of High Order Harmonics in the EUV Photon Energy Range, Opt. Express 19, 4346 (2011); A. Fleischer, O. Kfir, T. Diskin, P. Sidorenko, and O. Cohen, Spin 
Angular Momentum and Tunable Polarization in HighHarmonic Generation, Nat. Photonics 8, 543 (2014).

[23] B. Pfau, C. M. Günther, R. Könnecke, E. Guehrs, O. Hellwig, W. F. Schlotter, and S. Eisebitt, Magnetic Imaging at Linearly Polarized X-ray Sources, Opt. Express 18, 13608 (2010).

[24] K.-J. Kim, Circular Polarization with Crossed-Planar Undulators in High-Gain FELs, Nucl. Instrum. Methods Phys. Res., Sect. A 445, 329 (2000).

[25] Y. Ding and Z. Huang, Statistical Analysis of Crossed Undulator for Polarization Control in a Self-Amplified Spontaneous Emission Free Electron Laser, Phys. Rev. ST Accel. Beams 11, 030702 (2008).

[26] T. Wang et al., Femtosecond Single-Shot Imaging of Nanoscale Ferromagnetic Order in Co/Pd Multilayers Using Resonant X-ray Holography, Phys. Rev. Lett. 108, 267403 (2012).

[27] Haixiao Deng et al., Polarization Switching Demonstration Using Crossed-Planar Undulators in a Seeded Free-Electron Laser, Phys. Rev. ST Accel. Beams 17, 020704 (2014).

[28] M. Suzuki, Y. Inubushi, M. Yabashib, and T. Ishikawa, Polarization Control of an X-ray Free-Electron Laser with a Diamond Phase Retarder, J. Synchrotron Radiat. 21, 466 (2014).

[29] H.-D. Nuhn et al., in Proceedings of FEL2013, New York, NY, USA, 2013, http://accelconf.web.cern.ch/AccelConf/ fel2013/papers/tupso52.pdf.

[30] C. J. Bocchetta et al., FERMI@Elettra Conceptual Design Report, Technical Report No. ST/F-TN-07/12 (Sincrotrone Trieste, 2007).

[31] W. Barletta and C. Rizzuto, VUV and X-Ray Free-Electron Lasers: The Technology and Its Scientific Promise, Riv. Nuovo Cimento 29, 1 (2006).

[32] L. H. Yu, Generation of Intense UV Radiation by Subharmonically Seeded Single-Pass Free-Electron Lasers, Phys. Rev. A 44, 5178 (1991).

[33] E. Allaria et al., Highly Coherent and Stable Pulses from the FERMI Seeded Free-Electron Laser in the Extreme Ultraviolet, Nat. Photonics 6, 699 (2012).

[34] E. Allaria et al., Two-Stage Seeded Soft-X-ray FreeElectron Laser, Nat. Photonics 7, 913 (2013).

[35] T. Mazza et al., Determining the Polarization State of an Extreme Ultraviolet Free-Electron Laser Beam Using Atomic Circular Dichroism, Nat. Commun. 5, 3648 (2014).

[36] F. Capotondi et al., Coherent Imaging Using Seeded FreeElectron Laser Pulses with Variable Polarization: First Results and Research Opportunities, Rev. Sci. Instrum. 84, 051301 (2013).

[37] L. Müller et al., Ultrafast Dynamics of Magnetic Domain Structures Probed by Coherent Free-Electron Laser Light, Synchrotron Radiat. News 26, 27 (2013).

[38] C. von Korff Schmising, B. Pfau, M. Schneider, C. M. Günther, M. Giovannella, J. Perron, B. Vodungbo, L. Müller, F. Capotondi, E. Pedersoli, N. Mahne, J. Lüning, and S. Eisebitt, Imaging Ultrafast Demagnetization Dynamics After a Spatially Localized Optical Excitation, Phys. Rev. Lett. 112, 217203 (2014).

[39] Undulators, Wigglers and Their Applications, edited by H. Onuki and P. Elleaume (CRC Press, London, 2002).
[40] Y. K. Wu, N. A. Vinokurov, S. Mikhailov, J. Li, and V. Popov, High-Gain Lasing and Polarization Switch with a Distributed Optical-Klystron Free-Electron Laser, Phys. Rev. Lett. 96, 224801 (2006).

[41] M. Labat, M. Hosaka, M. Shimada, M. Katoh, and M. E. Couprie, Optimization of a Seeded Free-Electron Laser with Helical Undulators, Phys. Rev. Lett. 101, 164803 (2008).

[42] C. Spezzani, E. Allaria, M. Coreno, B. Diviacco, E. Ferrari, G. Geloni, E. Karantzoulis, B. Mahieu, M. Vento, and G. De Ninno, Coherent Light with Tunable Polarization from Single-Pass Free-Electron Lasers, Phys. Rev. Lett. 107, 084801 (2011).

[43] N. Čutić, F. Lindau, S. Thorin, S. Werin, J. Bahrdt, W. Eberhardt, K. Holldack, C. Erny, A. L'Huillier, and E. Mansten, Vacuum Ultraviolet Circularly Polarized Coherent Femtosecond Pulses from Laser Seeded Relativistic Electrons, Phys. Rev. ST Accel. Beams 14, 030706 (2011).

[44] S. Di Mitri et al., Design and Simulation Challenges for FERMI@elettra, Nucl. Instrum. Methods Phys. Res., Sect. A 608, 19 (2009).

[45] S. Di Mitri et al., in Advances in X-ray Free-Electron Lasers: Radiation Schemes, X-ray Optics, and Instrumentation, Vol. 8078, edited by T. Tschentscher and D. Cocco (SPIE, Prague, 2011), p. 807802.

[46] E. Allaria et al., Tunability Experiments at FERMI@Elettra Free Electron Laser, New J. Phys. 14, 113009 (2012).

[47] K. Halbach, Physical and Optical Properties of Rare Earth Cobalt Magnets, Nucl. Instrum. Methods Phys. Res. 187, 109 (1981).

[48] B. Diviacco, R. Bracco, D. Millo, and M. M. Musardo, in Proceedings of IPAC2011, Spain, 2011, http://accelconf .web.cern.ch/AccelConf/IPAC2011/papers/thpc164.pdf.

[49] M. Zangrando et al., The Photon Analysis, Delivery, and Reduction System at the FERMI@Elettra Free Electron Laser User Facility, Rev. Sci. Instrum. 80, 113110 (2009).

[50] L. Pivetta, G. Gaio, R. Passuello, and G. Scalamera, in Proceedings of ICALEPCS2011, France, 2011, http:// accelconf.web.cern.ch/AccelConf/icalepcs2011/papers/ thdaust03.pdf.

[51] L. Raimondi et al., Microfocusing of the FERMI@Elettra FEL Beam with a K-B Active Optics System: Spot Size Predictions by Application of the WISE Code, Nucl. Instrum. Methods Phys. Res., Sect. A 710, 131 (2013).

[52] P. Kirkpatrick and A. V. Baez, Formation of Optical Images by X-rays, J. Opt. Soc. Am. 38, 766 (1948).

[53] D. L. Windt, IMD-Software for Modeling the Optical Properties of Multilayer Films, Comput. Phys. 12, 360 (1998).

[54] Handbook of Optical Constants of Solids, Vol. 1, edited by E. D. Palik (Academic Press, New York, 1997); Handbook of Optical Constants of Solids, Vol. 2, edited by E. D. Palik (Academic Press, New York, 1997); Handbook of Optical Constants of Solids, Vol. 3, edited by E. D. Palik (Academic Press, New York, 1997); B. L. Henke, E. M. Gullikson, and J. C. Davis, X-ray Interactions: Photoabsorption, Scattering, Transmission, and Reflection at $E=50-30000 \mathrm{eV}$, $Z=1-92$, At. Data Nucl. Data Tables 54, 181 (1993); F. Schäfers and M. Krumrey, REFLEC: A Program to Calculate VUV/X-ray Optical Elements and Synchrotron Radiation Beamlines, Technischer Bericht TB 201/96 (1996) (BESSY, 1996). 
[55] F. Schäfers et al., Soft-X-ray Polarimeter with Multilayer Optics: Complete Analysis of the Polarization State of Light, Appl. Opt. 38, 4074 (1999).

[56] S. T. Manson and A. F. Starace, Photoelectron Angular Distributions: Energy Dependence for S Subshells, Rev. Mod. Phys. 54, 389 (1982).

[57] J. Viefhaus, F. Scholz, S. Deinert, L. Glaser, M. Ilchen, J. Seltmann, P. Walter, and F. Siewert, The Variable Polarization XUV Beamline p04 at PETRA III: Optics, Mechanics and Their Performance, Nucl. Instrum. Methods Phys. Res., Sect. A 710, 151 (2013).

[58] J. Viefhaus et al. (work in progress).

[59] V. Yu. Backman, S. V. Bobashev, and O. S. Vasyutinskii, Determination of Polarization of Vacuum-Ultraviolet Radiation by Fluorescence and Probe-Beam Techniques, Sol. Phys. 164, 397 (1996).

[60] C. J. Latimer, M. A. MacDonald, and P. Finetti, A New Method for Polarization Analysis in the VUV, J. Electron Spectrosc. Relat. Phenom. 101-103, 875 (1999).

[61] H. Elliott White, Introduction to Atomic Spectra (McGraw-Hill, New York, 1934).
[62] W. L. Wiese, M. W. Smith, and B. M. Glennon, NSRDS 4 Vol. 1 Atomic Transition Probabilities. Elements Hydrogen through Neon, NSRDS 4 (NBS, Washington DC, 1966).

[63] P. Finetti et al. (work in progress).

[64] C. Grazioli et al., CITIUS: An Infrared-Extreme Ultraviolet Light Source for Fundamental and Applied Ultrafast Science, Rev. Sci. Instrum. 85, 023104 (2014).

[65] A. Gaupp and M. Mast, First Experimental Experience with a VUV Polarimeter at BESSY, Rev. Sci. Instrum. 60, 2213 (1989).

[66] T. Koide, T. Shidara, M. Yuri, N. Kandaka, K. Yamaguchi, and H. Fukutani, Elliptical-Polarization Analyses of Synchrotron Radiation in the 5-80-eV Region with a Reflection Polarimeter, Nucl. Instrum. Methods Phys. Res., Sect. A 308, 635 (1991).

[67] G. De Ninno, B. Mahieu, E. Allaria, L. Giannessi, and S. Spampinati, Chirped Seeded Free-Electron Lasers: Self-Standing Light Sources for Two-Color PumpProbe Experiments, Phys. Rev. Lett. 110, 064801 (2013). 